\title{
Theoretical modelling of intermediate band solar cell materials based on metal-doped chalcopyrite compounds
}

\author{
P. Palacios ，K. Sánchez , J.C. Conesa , J.J. Fernández , P. Wahnón
}

\begin{abstract}
Electronic structure calculations are carried out for $\mathrm{CuGaS}_{2}$ partially substituted with $\mathrm{Ti}, \mathrm{V}, \mathrm{Cr}$ or $\mathrm{Mn}$ to ascertain if some of these systems could provide an intermediate band material able to give a high efficiency photovoltaic cell. Trends in electronic level positions are analyzed and more accurate advanced theory levels (exact exchange or Hubbard-type methods) are used in some cases. The Ti-substituted system seems more likely to yield an intermediate band material with the desired properties, and furthermore seems realizable from the thermodynamic point of view, while those with $\mathrm{Cr}$ and $\mathrm{Mn}$ might give half-metal structures with applications in spintronics.
\end{abstract}

Keywords: Chalcopyrite; Intermediate band materials; Electronic Structure; Thermodynamics

\section{Introduction}

Transition metal (TM)-substituted semiconductors in which the TM generates a new band in the host material band gap are receiving large attention in recent years, due to their potential for spintronics which arises from their having metallic character but with electron density of only one type of spin (majority or minority) at the Fermi level.

Another proposed application is the implementation of the intermediate band (IB) concept, postulated [1] as able to enhance the efficiency of photovoltaic (PV) cells. In this concept, an electron can be excited across a large energy gap $(\approx 2.0 \mathrm{eV})$ not only by one high energy photon but also by absorption of two lower energy photons, leading to an ideal solar energy conversion limit of $63.2 \%$, higher than the $41 \%$ of normal single-junction cells. For this, a moderately narrow IB located within the bandgap of the parent semiconductor and well separated of the valence and conduction bands of the latter is required. The IB must be partially filled (i.e. metallic), to allow good absorption in both transitions of interest. Transition metal-doped $\mathrm{MI}-\mathrm{V}$ materials have been the first candidates, proposed by us, for implementing this concept [2-4]; others derived from II-VI compounds have been also suggested [5].

More recently we have suggested that transition metal-doped chalcopyrites can be used as well to this aim [6]. Here we explore further this idea, computing with density functional theory (DFT) methods the electronic structures for a series of such compounds where some $\mathrm{Ga}$ atoms of the $\mathrm{CuGaS}_{2}$ chalcopyrite are substituted with different TMs of the first row, and addressing also the use of advanced DFT approaches beyond today's standard one (the generalized gradient approximation, GGA) to get reliable results.

\section{Models and methods}

The unit cell used in all calculations in this work has been constructed from the vectors (a,b,c) of the tetragonal chalcopyrite cell according to $\mathbf{a}^{\prime}=(\mathbf{a}+\mathbf{b}+\mathbf{c}) / 2, \mathbf{b}^{\prime}=(\mathbf{a}+\mathbf{b}-\mathbf{c}) / 2$ and $\mathbf{c}^{\prime}=\mathbf{b}-\mathbf{a}$ ) [6]. In the resulting 16 -atom cell one $\mathrm{Ga}$ atom is replaced by a transition metal $\mathrm{M}$; the obtained $\mathrm{Cu}_{4} \mathrm{MGa}_{3} \mathrm{~S}_{8}$ cell is then the primitive of a centered monoclinic lattice with $\mathrm{C} 2$ symmetry, where the shortest $M-M$ distance is $\approx 6.6 \AA$.

Spin-polarized DFT calculations at the GGA PW91 level were carried out with the plane-wave VASP code using PAW potentials [7]. The energy cutoff of the planewave expansion 
was $280 \mathrm{eV}$, we have checked that this is enough to get accurate results with PAW potentials. The Brillouin zone was sampled using $6 \times 6 \times 6$ Monkhorst-Pack grids with 112 irreducible k-points including the $I$ ' point. Unconstrained atomic relaxations were made with convergence tolerance of $0.01 \mathrm{eV} / \AA$ for atomic forces. Projections of densities of states (DOS) on spherical harmonics (to get orbital components) were obtained integrating inside atom-centered spheres having the standard atomic radii of the corresponding species. Energetic feasibility predictions were made for the candidate materials by comparing total GGA-level energies of the appropriate compounds.

GGA has known limitations for handling transition metal compounds having partially filled valence $3 \mathrm{~d}$ levels, which lead to important correlation effects. A deeper, more accurate study was thus made for the most interesting cases through inclusion of an effective on-site Coulomb parameter $U$ [8] (the Hubbard $\mathrm{GGA}+U$ approach) applied over the $3 \mathrm{~d}$ electrons of the corresponding transition metal.

Finally, the well-known problem that normal DFT (with or without $U$ corrections) has in predicting energy separations between filled and empty levels, due to an inaccurate handling of electron exchange, was addressed. Some calculations were thus made within the Exact Exchange (EXX) formalism, as implemented by us in the DFT code SIESTA, employing an atomic basis function representation [9]. Here spin polarization could not be used, and the correlation was not considered.

\section{Results}

\subsection{Electronic structure calculations at the GGA level}

After relaxing cell dimensions and atomic positions, no relevant variation of the cell volumes nor $\mathrm{M}-\mathrm{S}$ distances has been found for $\mathrm{V}, \mathrm{Cr}$ and $\mathrm{Mn}$. In the case of $\mathrm{Ti}$, a change in cell volume of $-0.61 \%$ and an average Ti-S bond distance of $2.35 \AA$ were obtained [6]. Highly spin polarized electronic structures were found, the number of unpaired spins per primitive unit cell in the four cases examined $(\mathrm{M}=\mathrm{Ti}, \mathrm{V}, \mathrm{Cr}$ and $\mathrm{Mn}$ ) being very close to 1, 2, 3 and 4 respectively. Fig. 1 shows the relevant DOS and projected DOS (PDOS) curves obtained. Comparison of the parent $\mathrm{CuGaS}_{2}$ DOS results with those of Msubstituted compound has been presented elsewhere for the $\mathrm{Ti}$ case [9].

The results can be interpreted considering that $\mathrm{M}$ is in a (formal) $\mathrm{M}^{3+}$ oxidation state, and that, in accordance with standard inorganic chemistry concepts, its partially filled $3 \mathrm{~d}$ shell is split by the nearly tetrahedral environment in two main manifolds: a lower one with two states, related to the $e$ symmetry species of purely tetrahedral symmetry, and an upper one with three levels corresponding to the $t_{2}$ species. The PDOS curves confirm in all cases (except for $\mathrm{Mn}$ ) the $\mathrm{M}^{3+}$ state, and show that the minority spin states with mainly M-3d character are always empty, all the electrons associated to the partially occupied $3 \mathrm{~d}$ shell being in majority spin states; i.e., the $3 \mathrm{~d}$ shell is fully spin-polarized. This corresponds to the high spin state in the typical inorganic chemistry description.
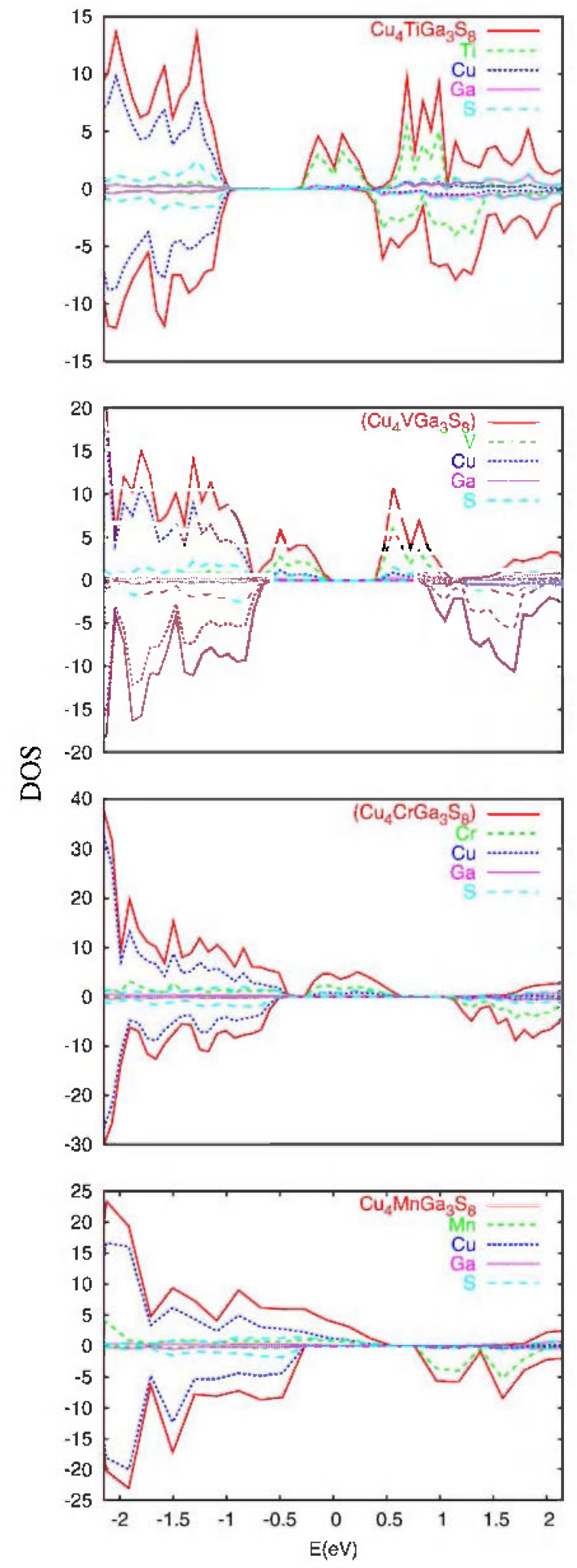

Fig. 1. DOS and PDOS curves obtained with GGA for metal-substituted $\mathrm{CuGaS}_{2}$ chalcopyrite for majority spin (upper part of the plots) and minority spin (lower part of the plots). The energy zero in abscissas marks the Fermi level.

Within the relevant occupied majority spin states, it can be seen that, in the $\mathrm{M}=\mathrm{Ti}$ case, the lower manifold (e-type) of metal-based levels appears mostly within the bandgap of the 
Transition metal 3d levels

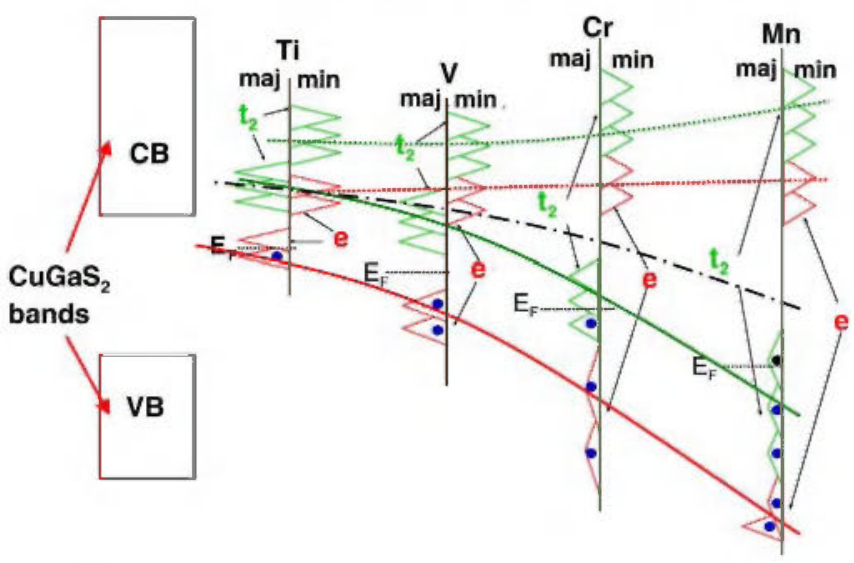

Fig. 2. Scheme of the positions, relative to the conduction and valence bands of the chalcopyrite host, of the $3 \mathrm{~d}$ levels of the transition metal substituting a $\mathrm{Ga}$ atom. The evolution of the e-type and $t_{2}$-type manifolds in both majority and minority spins ( full and dotted lines respect.) is marked, as well as the location of the Fermi level $\mathrm{E}_{\mathrm{F}}$, as a function of the $3 \mathrm{~d}$ levels filling by unpaired electrons (dots).

parent semiconductor, and only its upper part overlaps somewhat the conduction band $(\mathrm{CB})$ of the latter; it is (partially) occupied with one electron, being intersected by the Fermi level, so that the conditions for an IB of PV interest are nearly fulfilled. In the $\mathrm{M}=\mathrm{V}$ case, the e-type manifold has lowered in energy (due to the higher effective atomic charge), and although appearing largely within the main bandgap it overlaps the valence band (VB) of the chalcopyrite host; furthermore, it is completely electron-filled, being thus not useful for the PV IB concept.

In the $\mathrm{M}=\mathrm{Cr}$ case the $e$ manifold, lower in energy, is fully hybridized with the $\mathrm{VB}$, but the $t$, manifold appears isolated within the gap, and is partially occupied by one electron; it would seem in principle adequate for developing the PV IB scheme. Finally, in the $\mathrm{M}=\mathrm{Mn}$ case the $t$, manifold, partially filled, has lowered in energy so that a large overlap with the valence band occurs, and there is a significant contribution of

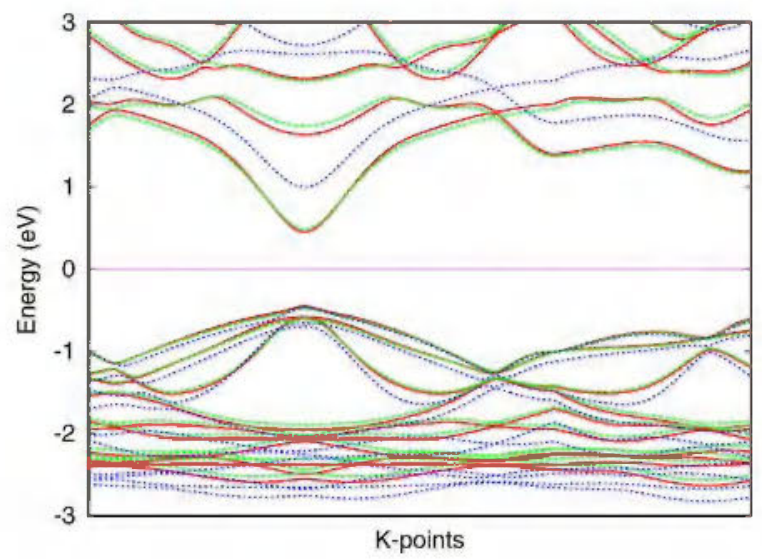

Fig. 3. Band structure comparison for $\mathrm{CuGaS}_{2}$ calculated using the SIESTA code with the approaches GGA (solid line) and EXX, with 88 (dashed line) and 120 (dots) Slater functions.

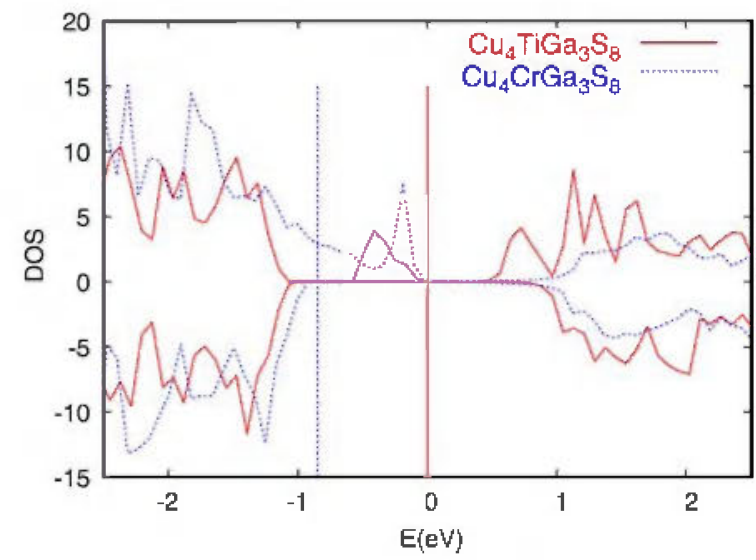

Fig. 4. DOS and PDOS curves obtained at the GGA $+U$ level for Ti- (solid line) and Cr-doped (dots) $\mathrm{CuGaS}_{2}$. Vertical lines mark the Fermi level for each system.

$\mathrm{Cu}$ and $\mathrm{S}$ to the states above the Fermi level, evidencing a deloealization of the hole which was initially in the $\mathrm{Mn}$ ion. This is unsuitable for the PV IB concept, but, as stressed in recent papers obtaining similar results for this system [10], could be interesting for spintronics as this system, like the much studied Mn:GaAs one, has according to these data half-metallic character.

The evolution of electronic levels shown by these results can be summarized in the scheme of Fig. 2. The splitting between majority and minority spins is seen to increase with atomic number within the series, due to the increasing number of unpaired electrons in this high spin situation (the situation would be reversed for the transition metals further to the right in the periodic table, as the $3 \mathrm{~d}$ shell becomes more than half filled). At the same time, the center of gravity of the occupied $3 d$ levels is also lowered due to the higher effective nuclear charge as said above. It is expected that this type of scheme may be useful to understand, and predict, the behavior of this kind of systems when studying other semiconductors (tetrahedral or not) substituted with different transition metals.

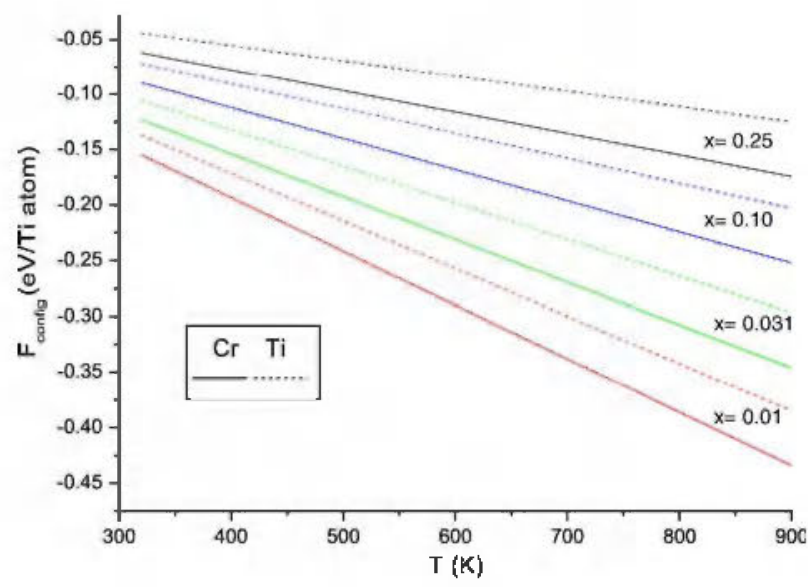

Fig. 5. Contribution of disorder entropy to the free energy of formation of Tiand $\mathrm{Cr}$-substituted $\mathrm{CuGaS}_{2}$ chalcopyrite. 


\subsection{Higher accuracy calculations}

The preceding results indicate that some metal-doped chalcopyrites are IB materials, but it must be recalled that standard DFT is not accurate in predicting band gaps in semiconductors. Indeed a similar calculation for $\mathrm{CuGaS}_{2}$ yields a gap of ca. $1.5 \mathrm{eV}$, while the experimental one is $2.4 \mathrm{eV}$. This could be remediated largely if a description of electron exchange interaction better than that available in normal DFT were applied. For this the EXX approach can be used. Indeed this was verified with our implementation of EXX in the SIESTA code, which gave satisfactory results both for III-V semiconductors [11] and for these doped with $\mathrm{Ti}$ [12]; in the latter case, isolation (and some natrowing) of an intermediate band could be verified.

For the systems examined here, application of this method is more difficult, due to the higher number of atoms and electrons in the cell (the full $3 \mathrm{~d}^{10}$ shell of $\mathrm{Cu}$ must be included), making the task very computer-costly. The capacity of this method to give for $\mathrm{CuGaS}_{2}$ a gap value closer to the experiment could at least be verified. Fig. 3 compares the results of two different EXX calculations (using bases of 88 and 120 Slater functions to represent the exchange potential) with a GGA calculation (the band edge positions and singular points yielded by this latter being quite similar to those found during our previous GGAVASP study on CuGaS2 [6], which validates the consideration here of the SIESTA calculation). When the biggest basis is used an increase of $0.564 \mathrm{eV}$ is found in the gap. The situation should improve with an optimized basis set, now under development; hopefully full EXX calculations will be feasible in the near future even for the metal-doped systems. Since the main effect of this method is to increase the separation between filled and empty levels, it should not change the relative position of the VB and IB from that given by the other DFT methods, and thus we can expect that in an exact calculation, and in the real solid, the overlap seen in Fig. 1 for the Ti-substituted case between the $\mathrm{CB}$ of the parent chalcopyrite and the metal-induced $e$-type band will disappear, so that the latter will become a real IB well isolated inside the gap.

Besides exchange, also correlation effects are not well reproduced by standard DFT, especially when $3 d$ electrons are present. To improve the prediction accuracy GGA $+U$ calculations were made in the potentially more interesting cases (those with $\mathrm{Ti}$ and $\mathrm{Cr}$, on the basis of the results above). $U$ values were taken from the literature: $5 \mathrm{eV}$ for $\mathrm{Cr}$ [13] and $2 \mathrm{eV}$ for Ti [14]. The results, presented in Fig. 4, show as expected a lowering and rise in energy of the filled and empty $3 \mathrm{~d}$ orbitals-based states, respectively. The effect is that Ti gives now clearly an IB separated from the CB and VB of the $\mathrm{CuGaS}_{2}$ host, while for $\mathrm{Cr}$ it is merged with the VB, making this system no longer valid for PV IB use (but does keep a half-metal character, so it could be useful for spintronics). For Ti, the IB found is now filled (the other $e$ state, empty, is pushed up to merge with the $\mathrm{CB}$ ); therefore hole doping will be required to make it partially filled as required for efficient PV IB operation. However, it must be noted that the $U$ values used, taken from literature, are fitted to compounds different from those studied here; further work to verify which values are suitable in our case is thus needed before the latter conclusions can be considered firm.

\subsection{Thermodynamics of compound formation}

The question can be now posed, whether these compounds, at least in the two cases more interesting for the PV IB concept, are realizable. A first indication comes from DFT calculations of total energy made for them and for the nearest simpler compounds from which they might form. For the $\mathrm{Cr}$ case one may consider the process

$$
\mathrm{CuCrS}_{2}+3 \mathrm{CuGaS}_{2} \rightarrow \mathrm{Cu}_{4} \mathrm{Ga}_{3} \mathrm{CrS}_{8}
$$

as $\mathrm{CuCrS}_{2}$ is a stable and known compound. For the Ti case, however, a CuTiS ${ }_{2}$ compound has not been reported. The closest one is $\mathrm{Cu}_{0.7} \mathrm{TiS}_{2}$ [15]. Thus as model of the Ti-doped chalcopyrite formation one can take the reaction

$$
3 \mathrm{CuGaS}_{2}+\mathrm{Cu}_{2 / 3} \mathrm{TiS}_{2}+1 / 3 \mathrm{Cu} \rightarrow \mathrm{Cu}_{4} \mathrm{Ga}_{3} \mathrm{TiS}_{8}
$$

DFT calculations at the GGA level give for these processes energy balances of respectively +0.82 and $+0.61 \mathrm{eV} / \mathrm{M}$ atom, i.e. the doped compound is unstable compared to the simpler ones. This is probably because $\mathrm{M}$ has in chalcopyrite tetrahedral coordination, less favorable for it than the octahedral one of these pure $\mathrm{Cu}-\mathrm{M}-\mathrm{S}$ compounds. However, as explained previously for the insertion of $\mathrm{Ti}$ in GaP and GaAs [4], this does not forbid the obtention of such compound material, at least for low (<5\%) M contents, since Mn insertion has similar energy cost and however has been experimentally achieved. Note also that tetrahedral $\mathrm{Ti}^{3+}$ has been detected in $\mathrm{ZnS}$ and other similar semiconductors [16].

A factor facilitating it can be the entropic contribution to free energy. Its vibrational part has not been computed, but its disorder component (configurational entropy) can be evaluated; its balance for the processes above is presented in Fig. 5. It depends on both temperature and $\mathrm{M}$ concentration (being different in the $\mathrm{Ti}$ and $\mathrm{Cr}$ cases because in $\mathrm{Cu}_{2 / 3} \mathrm{TiS}_{2}$ there is disorder in the population of the $\mathrm{Cu}$ sites, giving an entropy contribution, contrarily to the case of the Cr compound), and at high temperature and low concentration levels it can cancel a significant part of the positive unfavorable total energy balance. Thus these M-doped chalcopyrites are probably feasible in practice, at least for low substitution levels.

\section{Conclusions}

Electronic structures of $\mathrm{CuGaS}_{2}$ with Ga partially substituted by a transition metal $(\mathrm{Ti}, \mathrm{V}, \mathrm{Cr}, \mathrm{Mn}$ ) have been computed with DFT methods. The position and population of the new levels due to the metal can be analyzed based on inorganic chemistry concepts. The importance of using advanced DFT methods $(\mathrm{GGA}+U, \mathrm{EXX})$ for good evaluation of electronic structure is emphasized. The results indicate that the Ti-doped compound is the best candidate for realising the PV IB concept and seems feasible on thermodynamic grounds, while the Cr- 
and Mn- containing systems could be interesting for spintronics applications.

\section{Acknowledgements}

We acknowledge funding from project FULLSPECTRUM (contract. SES-CT-2003-502620) of the 6th European Framework Programme and from the Spanish National R\&D contract MAT2004-04631 and the project NUMANCIA (No. S-0505/ ENE/000310) funded by the Comunidad Autonoma de Madrid.

\section{References}

[1] A. Luque, A. Marti, Phys. Rev. Lett. 78 (1997) 5014

[2] P. Wahnon, C. Tablero, Phys. Rev., B 65 (2002) 165115.

[3] P. Wahnon, P. Palacios, J.J. Fernandez, C. Tablero, J. Mater. Sci. 40 (2005) 1383.

[4] P. Palacios, J.J. Fernandez, K. Sanchez, J.C. Conesa, P. Wahnon, Phys. Rev. B 73 (2006) 085206.

[5] K.M. Yu, W. Walukiewicz, J. Wu, W. Shan, J.W. Beeman, M.A. Scarpulla, O.D. Dubon, P. Becla, Phys. Rev. Lett. 91 (2003) 246403.
[6] P. Palacios, K. Sanchez, J.C. Conesa, P. Wahnon, Phys. Stat. Solidi, A Appl. Res. 203 (2006) 1395.

[7] G. Kresse, J. Hafner, Phys. Rev. B 47 (1993) RC558;

G. Kresse, J. Furthmüller, Phys. Rev. B 54 (1996) 11169 ;

G. Kresse, J. Joubert, Phys. Rev. B 59 (1999) 1758.

[8] S.L. Dudarev, G.A. Botton, S.Y. Savrasov, C.J. Humphreys, A.P. Sutton, Phys. Rev. B 57 (1998) 1505.

[9] J.M. Soler, E. Artacho, J.D. Gale, A. Garcia, J. Junquera, P. Ordejon, D. Sanchez-Portal, J. Phys., Condens. Matter 14 (2002) 2745.

[10] S. Picozzi, Y. Zhao, A. Freeman, B. Delley, Phys. Rev. B 66 (2002) 205206.

[11] J.J. Fernandez, C. Tablero, P. Wahnon, J. Chem. Phys. 120 (2004) 10780

[12] J.J. Fernandez, C. Tablero, P. Wahnon, Comput. Mater. Sci. 28 (2003) 2774 .

[13] A.I. Liechtenstein, V.I. Anisimov, J. Zaanen, Phys. Rev. B 52 (1995) R5467.

[14] A.I. Poteryaev, A.I. Lichenstein, G. Kotliar, Phys. Rev. Lett. 93 (2004) 086401.

[15] P. Palacios, P. Wahnon, S. Pizzinato, J.C. Conesa, J. Chem. Phys. 124 (2006) 014711.

[16] J. Dziesiaty, M.U. Lehr, P. Peka, A. Klimakow, S. Muller, H.J. Schulz, Eur. J. Phys. B 4 (1998) 269. 